\title{
Cloud inhomogeneity from MODIS
}

\author{
Lazaros Oreopoulos $^{1,2}$ and Robert F. Cahalan ${ }^{2}$
}

1. JCET-University of Maryland Baltimore County, Baltimore MD

2. Laboratory for Atmospheres, NASA-Goddard Space Flight Cente, Greenbelt, $M D$

Submitted to J. Climate, September 2004 


\begin{abstract}
Two full months (July 2003 and January 2004) of MODIS Atmosphere Level-3 data from the Terra and Aqua satellites are analyzed in order to characterize the horizontal variability of cloud optical thickness and water path at global scales. Various options to derive cloud variability parameters are discussed. The climatology of cloud inhomogeneity is built by first calculating daily parameter values at spatial scales of $1^{\circ} \times 1^{\circ}$, and then at zonal and global scales, followed by averaging over monthly time scales. Geographical, diurnal, and seasonal changes of inhomogeneity parameters are examined separately for the two cloud phases, and separately over land and ocean. We find that cloud inhomogeneity is weaker in summer than in winter, weaker over land than ocean for liquid clouds, weaker for local morning than local afternoon, about the same for liquid and ice clouds on a global scale, but with wider probability distribution functions (PDFs) and larger latitudinal variations for ice, and relatively insensitive to whether water path or optical thickness products are used. Typical mean values at hemispheric and global scales of the inhomogeneity parameter $v$ (roughly the mean over the standard deviation of water path or optical thickness), range from $\sim 2.5$ to 3 , while for the inhomogeneity parameter $\chi$ (the ratio of the logarithmic to linear mean) from $\sim 0.7$ to 0.8 . Values of $\chi$ for zonal averages can occasionally fall below 0.6 and for individual gridpoints below 0.5 . Our results demonstrate that MODIS is capable of revealing significant fluctuations in cloud horizontal inhomogenity and stress the need to model their global radiative effect in future studies.
\end{abstract}




\section{Introduction}

The non-linear dependence of solar and longwave radiation on cloud optical properties is a fundamental aspect of atmospheric radiative transfer and its importance for Earth's climate was noted many years ago (e.g., Harshvardhan and Randall, 1985). It is not then surprising that the detailed implications of this nonlinearity on cloud remote sensing and radiative fluxes have been studied vigorously in recent years. However, only a handful of these studies was of global scope, as far as we know (the observational study of Rossow et al., 2002 and the model-based studies of Oreopoulos et al., 2004 and Räisänen et al., 2004). The present study, while focusing only on a specific aspect of cloud heterogeneity, namely the horizontal fluctuation of cloud water path $W$ or optical thickness $\tau$ (hereafter sometimes referred to collectively as "cloud optical properties") is also of global scope. The studies of cloud water horizontal fluctuations that preceded Rossow et al. (2002) (hereafter RDC) provided an incomplete and often conflicting picture of their magnitude because they were based on limited number of scenes and retrievals from very different satellite instruments (Barker, 1996; Oreopoulos and Davies, 1998a; Pincus et al., 1999). In the following we will demonstrate that, as with the International Satellite Cloud Climatology Project (ISCCP) products of RDC, higher level cloud products from the Moderate Resolution Imaging Spectroradiometer (MODIS) instrument aboard the Terra and Aqua satellites can be used for a thorough study of horizontal cloud heterogeneity.

Knowledge of the geographical and seasonal distribution of observed cloud inhomogeneity is essential in any effort to make it a diagnosed or predicted quantity in Large Scale Models (LSMs). One may of course ask why there is need for LSMs to add another variable to keep track of in their simulations. The short answer is that by doing so 
they will be able to more accurately represent physical processes involving clouds. These include both microphysical processes related to cloud and rain formation (e.g. Jakob and Klein, 1999), but also, processes that mostly interest the authors of this paper, namely interactions with solar and thermal radiation. The need to adopt a radiative scheme which implicitly accounts for cloud horizontal inhomogeneity arises from the fact that planeparallel homogeneous (PPH) radiation flux calculations, i.e., calculations where only the mean cloud optical properties of the gridbox are used, often exhibit significant errors compared to the more accurate Independent Column Approximation (ICA) calculations where averages of plane parallel calculations on individual cloudy columns (currently not explicitly resolved by LSMs) or integrals over the probability density function (PDF) of optical properties are taken. These systematic errors, frequently referred to as plane parallel biases (Cahalan et al. 1994), stem from the nonlinear dependence of both shortwave (SW) and longwave (LW) fluxes on cloud optical properties. The magnitude of the errors depends on solar geometry (for SW), the average amount of cloud water, cloud vertical overlap, and the degree of cloud heterogeneity. The SW albedo error, for example, was found from theoretical and observational studies to have a range of 0.025 to 0.3 (in a literature survey by RDC), suggesting a very substantial impact on the energy budget. It is quite obvious that LSM modelers would not want their efforts to simulate correct mean distributions of cloud properties be hampered by their inability to produce realistic radiation budgets. If the goal is to develop schemes that account either implicitly or explicitly for the subgrid cloud water heterogeneity (e.g. Tompkins, 2002), it is only logical to assume that global descriptions of the magnitude of the heterogeneity based on observations will aid the development of the relevant algorithms. 
Our paper, whose aim is to provide significant elements of this global description, is organized as follows. First, we present in section 2 the various parameters that have in recent years been used as descriptors of cloud water horizontal variability. Then in section 3 we discuss which of these parameters can be obtained from the MODIS dataset, which parameter we chose to put most emphasis on in this study and why, how retrieved values of the parameters are averaged to build the climatology, and what the limitations of the MODIS dataset are. Section 4 is the heart of the paper and is dedicated to results showing various aspects of the global picture of cloud horizontal inhomogeneity: differences between water and ice clouds, morning and afternoon clouds, marine and continental clouds, winter and summer clouds, etc. An overview of the results, their meaning for the Earth's radiation budget, and ideas on how they can be used in future studies is offered in the final section.

\section{Measures of horizontal cloud heterogeneity}

Several parameters have been suggested in the literature as decriptors of horizontal variability in cloud water or optical thickness. Whether used as a diagnostic quantity of cloud horizontal heterogeneity or as input in an algorithm that accounts for the variability in a modeling application, each parameter has its advantages and disadvantages. When inferred from space observations, each also has different sensitivities to uncertainties in the satellite measurements used to derive it. One of the benefits of having several measures of cloud horizontal inhomogeneity available is that comparisons among them can, in some occasions, reveal information on characteristics of the optical property distribution within a region (in our case, as discussed later, a gridpoint of the Level-3 
MODIS cloud products) that could not have been inferred by using only a single inhomogeneity measure (see brief discussion at the end of the section). A short overview of the various parameters that have been used to quantitatively describe cloud horizontal inhomogeneity is provided below.

The inhomogeneity parameter $\chi$ first introduced by Cahalan et al. (1994) is defined as the ratio of the logarithmically averaged to the linearly averaged optical thickness of a region:

$$
\chi=\frac{e^{\overline{\ln \tau}}}{\bar{\tau}} \quad 0<\chi \leq 1
$$

with

$$
\bar{\tau}=\int \tau p(\tau) \mathrm{d}(\tau)
$$

and $\overline{\ln \tau}$ defined similarly to $\bar{\tau}$ :

$$
\overline{\ln \tau}=\int \ln \tau p(\tau) \mathrm{d} \tau
$$

$p(\tau)$ is the probability density function (PDF) of optical thickness $\tau$. The inception of $\chi$ was inspired by the fact that the reflected solar flux is a linear function of the logarithm of optical thickness for a wide range of optical thicknesses. Thus, the product $\chi \bar{\tau}$ is the logarithmically averaged optical thickness and provides a better regional albedo estimate 
than $\bar{\tau}$ when used as input radiative transfer calculations. Oreopoulos and Davies (1998b) compared $\chi$ with $\chi_{0}$ :

$$
\chi_{0}=\frac{\hat{\boldsymbol{\tau}}}{\overline{\boldsymbol{\tau}}}=\frac{\mathrm{R}^{-1}\left(R_{\mathrm{ICA}}\right)}{\overline{\boldsymbol{\tau}}} \quad 0<\chi_{0} \leq 1
$$

i.e., the ratio of the radiatively averaged optical thickness (the optical thickness that corresponds to the average reflected radiation of a region, possibly also integrated over the solar zenith angle) to the linearly averaged optical thickness. The inhomogeneity parameter $\varepsilon$ used by RDC in the cloud heterogeneity climatology from ISCCP is simply $1-\chi_{0}$. All these parameters can be obtained from radiometric observations such as those from MODIS as explained in the following. It must be noted however, that derivation of $\chi_{0}$ or $\varepsilon$ requires forward radiative transfer calculations to obtain $R_{\mathrm{ICA}}$, and will not be further discussed in this paper.

A different class of cloud horizontal inhomogeneity measures is related to theoretical distributions that have been found to be good approximations of actual distributions of cloud optical properties. For example, $v$, the shape parameter of a gamma distribution, can be derived from the ratio of mean to standard deviation of the distribution, in which case it is said that it is derived from the Method of Moments (MOM):

$$
v_{M O M}=\left(\frac{\bar{\tau}}{\sigma_{\tau}}\right)^{2}
$$


Within the context of the gamma distribution $v$ can also be estimated from the Maximum Likelihood Estimate (MLE) method (Wilks, 1995) which is less sensitive to outliers than MOM (Oreopoulos and Davies 1998b):

$$
\begin{aligned}
& v_{M L E}=\frac{1+\sqrt{1+4 y / 3}}{4 y} \\
& y=\ln \bar{\tau}-\overline{\ln \tau}
\end{aligned}
$$

Since $\tau$ distributions have also been approximated by lognormal distributions (Cahalan et al., 1994; Oreopoulos and Davies, 1998b), other useful inhomogeneity measures are the standard deviation of $\ln \tau$ or the ratio of mean to standard deviation of $\ln \tau$. The former has been used in Cahalan et al. (1994) and in Cahalan et al. (1995) to quantify variability of marine stratocumulus, while the latter has not been explored extensively, and will not be further discussed here either. $v$ (of both the MOM and MLE variety) can in principal also be inferred from satellite observations, and therefore from MODIS as well, and the exact way this can be done will be given in the next section.

As noted above, the various measures of inhomogeneity have their advantages and disadvantages as descriptors of cloud variability. Comparisons among them can give quick glimpses of the $\tau$ or $W$ distribution properties from which they were derived. For example, large deviations of $\chi$ from $\chi_{0}$ are found for distributions that are very wide, e.g., consisting of few very thick convective clouds mixed with very thin cirrus clouds as is common in the Tropics (RDC); large discrepancies between $v$ from MOM and MLE is indicative of ill-behaved optical thickness distributions, e.g., distributions with a small 
number of extremely large values that produces too small MOM values of $v$ for the resulting gamma distribution to be a good fit. In general, as RDC pointed out, the problem with inhomogeneity measures that involve calculations of the second moment of the distribution is that there may be convergence problems in the case of highly skewed or multimodal distributions and in situations of limited sample population (e.g. from daily histograms in regions with low cloud cover). As will be explained in the next section, the selection of the inhomogeneity parameters for this particular study is based on other criteria as well, such as the method of analysis, and specific characteristics of the MODIS Level-3 (L-3) dataset which is our exclusive source of optical property information.

\section{The MODIS dataset}

\section{a. Analysis method}

For the analysis in this paper we rely on L-3 (Global Gridded) MODIS cloud products. The cloud product monitors the physical and radiative properties of clouds including cloud particle phase (ice vs. liquid water), effective cloud particle radius, cloud optical thickness, cloud top temperature, cloud top height, effective emissivity, and cloud fraction under both daytime and nighttime conditions. There are three L-3 MODIS cloud products collected from two platforms, Terra and Aqua. Each of the L-3 products contain statistics generated from the Level-2 (Orbital Swath) products. Statistics are summarized over a $1^{\circ} \times 1^{\circ}$ global grid for daily (D3), eight-day (E3), and monthly (M3) temporal periods. In this study we only use D3 data. Statistics for a given derived quantity or SDS might include: simple (mean, minimum, maximum, standard deviation) statistics; parameters of normal and lognormal distributions; fraction of pixels that satisfy some 
condition (e.g. cloudy, clear); histograms of the quantity within each grid box; histograms of the confidence placed in the retrieved quantity; histograms and/or regressions derived from comparing one science parameter to another; statistics computed for a subset that satisfies some condition (King et al., 2003).

All these statistics are computed by subsampling pixel-level (Level-2) values every $5^{\text {th }}$ pixel along both spatial directions (King et al., 2003). Thus the cloud statistics for an overcast $1^{\circ} \times 1^{\circ}$ cell around the equator come from about $\sim 480$ pixels instead of the $\sim 120001-\mathrm{km}$ pixels that exist originally within the cell. Oreopoulos (2004) examined whether this subsampling has distorting effects on the Level-3 moments of optical thickness and the inhomogeneity parameters $\chi$ and $v$. He found that optical thickness subsampling errors were of random nature, as expected, and could therefore be supressed by taking averages over a number of regions. Since, as we describe below, we do a fair amount of averaging, both temporal (monthly scales) and spatial (zonal and global averages of the monthly means) the subsampling errors should be quite small. For example, zonal monthly percentage errors due to subsampling are at most times below $2 \%$ for $v_{\mathrm{MOM}}$. Errors for $v_{\mathrm{MLE}}$ and $\chi$ are even smaller since they depend on first moment quantities ( $\bar{\tau}$ and $\overline{\ln \tau}$ ) which are less sensitive to subsampling than $\sigma_{\tau}$. Oreopoulos (2004) found that $\chi$ exhibited the greatest resilience to subsampling, and this is one of the main reasons it is given maximum exposure in the results presented later. Other reasons for choosing to concentrate on $\chi$ is that it can be more easily compared with $\varepsilon$ of RDC and that its maximum assumed value of one facilitates averaging, in contrast to $v$ whose high values are unbounded. 
Two months (July 2003 and January 2004) of D3 data for both the Terra and Aqua platforms are used in this study. This data is freely available worldwide from the NASAGoddard Distributed Active Archive Center (DAAC) at http://daac.gsfc.nasa.gov.

In principle, estimates of all the cloud inhomogeneity parameters can be made using MODIS L-3 histograms for either $W$ or $\tau$. Differences in histogram discretization (see below) would most likely create differences in the values of the inhomogeneity parameters calculated for $\tau$ and $W$ even if cloud particle size remained constant within the $1^{\circ} \times 1^{\circ}$ gridpoint. The fact that particle size also varies spatially, contributes, of course, to additional differences. We opt for calculating inhomogeneity parameters from $\tau$ products (which are more directly linked to the radiative impact of cloud inhomogeneity) rather than $W$ products, except when we are specifically interested in examinining their differences (e.g. subsection 4b). The choice of the appropriate variable for assessing cloud inhomogeneity is not necessarily a trivial issue since it pertains to how cloud variability would potentially be represented in LSMs: if variability is prescribed or parameterized, observed $\tau$ inhomogeneity parameters should probably be used; if on the other hand it is predicted from the fundamental prognostic equations of a cloud scheme (e.g. Tompkins, 2002), prognosed values should be validated by comparing to observed $W$ inhomogeneity parameters. Below we describe in more detail how the inhomogeneity parameters are derived from the MODIS L-3 data.

The moments needed for the estimation of the inhomogeneity parameters, namely the mean of quantity $q(\tau$ or $W$ ), the mean of the logarithm (only for $\tau$ ), and the variance at a gridpoint $(m, n)$ for a given day $l$ are distinct SDS products included the D3 dataset, and can be therefore used directly. Alternatively, the moments can be calculated from the 
unnormalized frequency of occurrence $N_{\mathrm{j}}^{\prime}(m, n)$ of the SDS histograms, using the following expressions:

$$
\begin{aligned}
& \bar{q}_{l}(m, n)=\frac{\sum_{j=1}^{J} N_{j}^{l}(m, n) q_{j}}{\sum_{j=1}^{J} N_{j}^{l}(m, n)} \\
& \overline{\ln q_{l}(m, n)}=\frac{\sum_{j=1}^{J} N_{j}^{l}(m, n) \ln q_{j}}{\sum_{j=1}^{J} N_{j}^{l}(m, n)} \\
& \sigma_{q_{l}}^{2}(m, n)=\frac{\sum_{j=1}^{J} N_{j}^{l}(m, n)\left[q_{j}-\bar{q}_{l}(m, n)\right]^{2}}{\sum_{j=1}^{J} N_{j}^{l}(m, n)}
\end{aligned}
$$

In the above, $J$ is the number of bins in the histogram and $q_{\mathrm{j}}$ is the center value of the bin. For the D3 histograms of $\tau$ (as in their E3 and M3 counterparts) $J=45$ for liquid phase clouds and $J=30$ for ice clouds; the $W$ histograms are more sparsely resolved with $J=14$ bins for liquid phase clouds and $J=15$ for ice clouds. Note that eq. (8) is the only way to calculate the mean logarithm of $W$ (there is no separate SDS for this quantity).

The inhomogeneity measures obtained from the MODIS D3 histograms convey information mainly on spatial cloud variability since most regions are viewed only once by each satellite during daylight hours (this is less true for high latitudes where significant orbit overlap takes place). Contrast this with the E3 (M3) histograms of the $1^{\circ} \times 1^{\circ}$ regions which contain both the spatial and temporal variability realized during the 
eight-day period (month), and which are therefore wider than the D3 histograms. In this work we are mostly interested in spatial cloud variability because it is the most relevant for modeling applications: LSMs do indeed produce temporally varying clouds, but not clouds that vary spatially within a gridbox. For the purposes of a cloud inhomogeneity climatology, the relevant quantities will then be mean monthly values of inhomogeneity parameters derived by averaging the daily values obtained from the D3 SDS moments or the D3 (daily) histograms. Thus, for a $1^{\circ} \times 1^{\circ}$ region $(m, n)$ in the $m$ th meridional and $n$th latitudinal zone, the mean monthly value of the variability parameter $\chi$ will be given by:

$$
\bar{\chi}^{C}(m, n)=\frac{\sum_{l=1}^{L} C_{l}(m, n) \chi_{l}(m, n)}{\sum_{l=1}^{L} C_{l}(m, n)}
$$

where $L$ is the number of days for which it was possible to calculate $\chi_{1}(m, n)$, and $C_{\mathrm{l}}(m, n)$ is the cloud fraction for day $l$ (also a D3 SDS given separately for each phase) and region $(m, n)$. Eq. (10) gives thus a weighted (by cloud fraction) monthly mean value for the inhomogeneity parameter $\chi$ of a single gridpoint. Similar expressions are used to obtain the mean monthly values of $v$ for each $1^{\circ} \times 1^{\circ}$ region.

Spatial averaging of the inhomogeneity parameters is also performed. Zonal averages of $\chi($ similar for $v$ ) for a particular day $l$ are given by: 


$$
\left\langle\chi_{l}^{c}(n)\right\rangle=\frac{\sum_{m=1}^{M} C_{l}(m, n) \chi_{l}(m, n)}{\sum_{m=1}^{M} C_{l}(m, n)}
$$

where $M$ is the number of $1^{\circ} \times 1^{\circ}$ gridpoints in a certain latitude zone for which a value of $\chi_{1}(m, n)$ was calculated from eq. (1). The monthly zonal averages can then be obtained by simple arithmetic averaging of the daily zonal averages. Similarly, global averages can be obtained for a single day or an entire month. For a single day:

$$
\tilde{\chi}_{l}^{C}=\frac{\sum_{n=1}^{N} \sum_{m=1}^{M} w(n) C_{l}(m, n) \chi_{l}(m, n)}{\sum_{n=1}^{N} \sum_{m=1}^{M} w(n) C_{l}(m, n)}
$$

where $w(n)=\cos [\operatorname{lat}(n)]$ is the weight for each latitude zone calculated as the cosine at the center of the latitude zone, and $N$ is the number of latitude zones. The monthly global averages were calculated from arithmetically averaging the results of eq. (12). For the monthly zonal and global calculations it is assumed that day-to-day zonal and global variations of cloud fraction are small.

It must be pointed out that the cloud fraction used in eq. (10), (11), and (12) is not the cloud fraction of the MODIS cloud mask algorithm, but rather the cloud fraction calculated from the number of pixels for which the cloud retrieval algorithm was able to complete a successful retrieval of cloud optical properties. This cloud fraction is somewhat lower than the cloud fraction from the mask algorithm, but may be closer to 
the true cloud fraction, since the inability of the retrieval algorithm to match the observed and theoretical radiances for a particular vector of cloud property parameters is often indication that the pixel has been misclassified by the masking algorithm. The inhomogeneity parameters were calculated separately for each phase, even though calculations for all clouds combined are also possible (not further discussed or shown). While processing the data we discovered that retrieved optical thicknesses for ice clouds over land never assumed values below 1 . This was traced back to a programming error in the retrieval algorithm and will be corrected in future releases of the cloud products (Platnick, 2004, personal communication). This error is expected to result in slight underestimates of ice cloud variability over land, and unintentionally draws our attention to the limitations of cloud retrievals over land (can we really accurately retrieve optical thicknesses below 1?). No estimates of inhomogeneity parameters were pursued for the "undetermined" cloud phase (the cloud phase could not be unambiguously determined, but retrievals were performed with look-up tables for liquid clouds).

From eq. (5) and (6) it becomes obvious that $v$ can grow very large without bounds (e.g., as $\sigma_{\tau}$ gets progressively smaller). This occurs more frequently for $v_{\text {MOM }}$ and may take place for example when there are only very few cloudy pixels clustered in only a small area of the $1^{\circ} \times 1^{\circ}$ region or when indeed the cloud field appears very homogeneous to the MODIS radiometer. The extremely large values of $v$ of such cases, even if occuring relatively rarely, and despite their low weight in small cloud fraction cases, can have undesirable distorting effects on the temporal and spatial averages. To minimize the impact of these extreme values on spatial averages, two actions are taken: a) we consider only regions where cloud fraction exceeds 0.1 ; and b) we set an upper value of 10 for $v$ in 
the rather infrequent cases where the estimated value is larger. These two restrictions lead to some underestimates of $v$ (overestimates of variability). For consistency, only regions with cloud fraction greater than 0.1 are also used in the spatial averaging of $\chi$, even though the averages for this parameter will not suffer as much by distorting contributions from regions of weak inhomogeneity. As mentioned before, this is one of the reasons $\chi$ is so prominent in our analysis.

The issue that remains to be addressed is whether the inhomogeneity parameters of a gridpoint should be calculated from the moments of $\tau$ derived from the histograms using eq. (7-9), or from the moments provided as distinct SDS's by the MODIS L-3 aggregation algorithm. As noted before this choice does not exist for the mean logarithm of $W$ which is not provided as a L-3 SDS and must be therefore derived from eq. (8) (this affects the estimation of $\chi$ and $\left.v_{\mathrm{MLE}}\right)$. In general, the results obtained from (7-9) and directly from the L-3 SDS's are not identical. We will compare global values for both calculation methods in the next section. The advantage of using the histograms is that the results are self-consistent and cannot yield unphysical values of the inhomogeneity measures. For example, estimates of daily values of $\chi$ from (1) by using the linear and logarithmic means of $\tau$ as distincts SDS products, occasionally result in values that are slightly greater than 1 (when this happens we set $\chi=1$ ). The disadvantage of histograms is the inherent limitations of discretization which are accentuated for a small number of points (retrievals) in a gridpoint (e.g., small cloud fraction) and for higher order moment calculations (variance estimates from a histogram are less accurate than means). Since we consider the shortcoming of coarse discretization to have greater impact, we decided to use the SDS moments of D3 when they were available, and to use the histograms only 
when the desired quantity (e.g., mean logarithm of $W$ ) was not available as a distinct SDS.

\section{b. MODIS limitations}

MODIS can "see" only the total (integrated) optical thickness of one or more cloudy layers. If cloud variability were to be used as an input to the Column Radiation Model of an LSM, what would actually be most likely required would be the vertical profile of cloud variability. This cannot be provided by MODIS (which is not even providing the vertical profile of mean cloud optical thickness). In the Tropics, for example, highly variable convective towers may be covered by thick but relatively homogeneous cirrus anvils. Another issue is "real" vs. "apparent" cloud inomogeneity due to $3 \mathrm{D}$ radiative transfer effects inherent in MODIS observations. In the mid-latitudes, for instance, relatively homogeneous clouds of storm systems may look more inhomogeneous due to shadowing and side illumination effects not accounted for by the plane-parallel retrievals (Oreopoulos et al., 2000). In the first case MODIS may underestimate the variability of convective clouds, in the second case it may overestimate the variability of stratiform clouds. One can actually discover a lot about cloud variability by looking for systematic expressions of these MODIS limitations: it may be possible, for example, to learn a great deal about 3D effects on cloud retrievals, and therefore apparent cloud inhomogeneity, from intercomparing results among regions with climatologically similar single-layer clouds, but observed under different illumination conditions. Such issues will be given proper attention in a future study. 


\section{MODIS inhomogeneity climatology}

The following subsections examine various aspects of cloud horizontal inhomogeneity as obtained from the MODIS Level-3 cloud products. The analysis and discussion is by no means the ultimate word on what can be potentially accomplished with the MODIS dataset, but can be viewed as a blueprint for building more extensive multi-year cloud inhomogeneity climatologies from this particular source of cloud observations.

\section{a. Inhomogeneity parameter comparison}

It is natural to start the analysis of inhomogeneity by discussing the sensitivity of inhomogeneity parameters to the choice of calculation method (i.e., SDS moment-based vs. histogram-based, as discussed previously). This presentation is limited to global averages only.

Figure 1 shows the global mean values of $v_{\mathrm{MOM}}, v_{\mathrm{MLE}}, \chi$ for liquid phase and ice clouds from moment-based (index "1") histogram-based (index "2") estimates. These are values from the Terra platform for July ' 03 and are calculated using eq. (12) for $\chi$ and its exact counterparts for $v_{\mathrm{MOM}}$ and $v_{\mathrm{MLE}}$, and then calculating the monthly average from the daily values. Estimates are shown for both optical thickness and water path (WP legend in the figure) but discussion of these $\tau-W$ inhomogeneity differences is left for the next subsection. Also shown are estimates based on "QA" (Quality Assurance) moments, i.e., moments calculated using the QA quality flag values as weight (Platnick et al., 2003). The results of Fig. 1 indicate that the moment-based and histogram-based methods give similar global results with the histogram-based method yielding lower values for $v$. This 
difference is more pronounced when parameter calculations are based on $W$ statistics. However, using QA statistics seems to have a greater impact, with global cloud inhomogeneity apparently decreasing (parameter values increasing). This is because of the lower weight assigned to dubious retrievals of cloud optical thickness whose values likely fall close to the extrema of the $\tau$ value range. Since we do not use QA statistics in any of the calculations shown in the remainder of the paper, it is possible that we err slightly on the side of overestimating cloud heterogeneity.

It would be useful to obtain some empirical relationships to crudely convert values of any one of the three inhomogeneity measures $\chi, v_{\text {MOM }}$ and $v_{\text {MLE }}$ to values for the other two. The relationships are in general different from gridpoint to gridpoint since they depend on the details of the histogram shape, but in this section we will assume that regression results for zonal monthly averages (the most widely presented results of this paper) will be of sufficient quality for the type of parameterizations that could potentially be used in LSMs. Fig. 2, shows that the relationship between and $\chi$ and $v_{\text {MLE }}$ can be approximated by an exponential function for zonal averages, even though for individual gridpoints (i.e., before temporal and spatial averaging) they are related via eq. (6) since $y=-\ln \chi$. A similar exponential fit (albeit with a lesser quality of fit) can be applied to

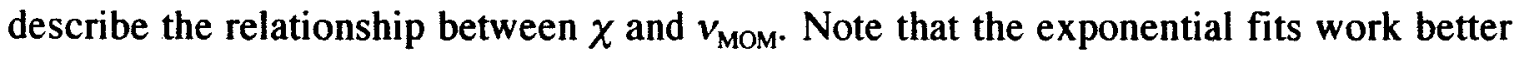
for the liquid clouds than the ice-phase clouds. Table 1 shows the approximate conversion rules from $\chi$ to $v_{\mathrm{MLE}}$ and $v_{\mathrm{MOM}}$ for zonal values; for other values of $\chi$, interpolation or direct application of the regression equations (given in Fig. 2) can be used. This table is then a rough guide for back-of-the-envelope conversions of the zonal results shown later for $\chi$ to zonal results for $v$. 


\section{b. Inhomogeneity from $\tau v$ s. inhomogeneity from $W$}

MODIS provides the opportunity to compare inhomogeneity parameters derived from $W$ and $\tau$ statistics. Global monthly values can be compared in Fig. 1 for July'03 and Terra retrievals. For $\chi$, liquid clouds appear slightly less homogeneous for $W$-based estimates while the opposite is observed for ice clouds, but the differences are quite small; for $v_{\text {MLE }}$ clouds appear slightly more heterogeneous for $W$-based estimates for both liquid and ice clouds; for $v_{\text {MOM }}, W$-based estimates are possible from both the SDS moments and the $W$ histograms and the results are inconsistent: clouds appear slightly (liquid) or significantly (ice) more homogeneous for moment-based parameter estimates from $W$ and less homogeneous for histogram-based parameter estimates from $W$. These results hence do not yield a conclusive picture on whether cloud particle variations work in the direction of enhancing or dampening cloud variability. The changes they bring about are not dramatic enough to stand out on a global scale and to overpower the impact of errors and approximations manifesting themselves in the calculations.

Figure 3 showing the monthly zonal averages of $\chi$ values obtained by averaging the daily values of eq. (11) for July'03/Jan'04 Terra liquid and ice clouds, and may shed a bit more light on the issue at hand. The difference between $\tau$-based and $W$-based values (both obtained from the histogram method) is greater for liquid than ice clouds and is consistent with the global values of the top panel of Fig. 1: particle size variations tend to reduce variability for liquid clouds (as in Räisänen et al., 2003) and increase it for ice clouds. The differences are stronger at low latitudes and somewhat stronger in January for liquid clouds and in July for ice clouds. Still, extended regions of agreement between both estimates can be seen in the midlatitudes for clouds of both phases. In general, these 
results show that not too drastically different conclusions about cloud inhomogeneity will be inferred regardless of whether one chooses to process optical thickness or water path products. Because optical thickness statistics are more complete and the histograms better resolved we work mostly with this optical property. Moreover, $W$ is not a directly retrieved quantity but a byproduct of combining $\tau$ and effective radius retrievals. This on one hand adds additional insight on the nature of cloud horizontal variability, but on the other hand burdens the estimates of the inhomogeneity parameters with additional uncertainties.

\section{c. Inhomogeneity of liquid clouds vs. inhomogeneity of ice clouds}

One of the MODIS cloud products is cloud thermodynamic phase. Possible outcomes of the cloud themodynamic phase identification algorithm are "uncertain phase", "mixed phase", "ice" or "liquid water" (Platnick et al., 2003). In this subsection we focus on the differences in inhomogeneity between the two "unambiguously" defined thermodynamic phases, i.e., "ice" and "liquid water". Some of the results that have been shown so far have depicted values of the inhomogeneity parameters separately for these two phases, and in this subsection we will revisit and discuss those findings.

Figure 1 shows that the global averages of the inhomogeneity parameters for July are similar for water and ice clouds, with only slightly smaller values (i.e., indicating slightly larger heterogeneity) for ice clouds. Figure 4 reveals that this near equality is the result of some latitudinal cancellations. In July, ice clouds tend to be more heterogeneous in the Tropics, northern subtropics and northern polar regions (where retrievals are less reliable). There are extensive mid-latitude regions in both the Northern and Southern 
hemisphere where the values of $\chi$ for both types of clouds are almost identical. In contrast, for January ice clouds seem to be more homogeneous than liquid clouds in most of the globe except the zone near the equator, north of $40^{\circ} \mathrm{N}$, and the high latitudes of the Southern hemisphere (again an area of less reliable retrievals). Another standout feature of Fig. 4 is that the latitudinal variability of the inhomogeneity parameters is more pronounced for ice than water clouds. A possible interpretation is that ice cloud morphology and mean optical properties are quite different among ice clouds associated with mid-latitude weather systems (some of which may be of mixed phase), thick cirrus anvils/deep convective towers that occur frequently in equatorial regions, and relatively thin cirrus clouds blanketing extensive regions without distinct geographic preference.

Further supporting this interpretation is Fig. 5 which shows histograms of $\chi$ derived from the monthly-averaged values of eq. (10) for Terra data. The histograms for ice clouds are broader than their water counterparts: the frequency of occurrence tends to be lower around the mode and higher around the front and end tail. In other words, ice clouds are more likely to be either extremely homogeneous or extremely heterogeneous compared to liquid clouds.

\section{d. Inhomogeneity from Terra vs. inhomogeneity from Aqua}

Although the full diurnal cycle cannot be resolved with the two sun-synchronous satellite platforms (Terra and Aqua) that carry MODIS, we can nevertheless study whether there are distinct differences in cloud heterogeneity at global scales before and after local noon. Figure 6 shows global-land and global-ocean averages of $\chi$, in a manner similar to Fig. 1 , for both water (top) and ice (bottom) clouds. Aqua (crossing the equator at $\sim 1: 30 \mathrm{pm}$ 
local time) has in general smaller values of $\chi$ (larger apparent cloud heterogeneity) than Terra (with an approximate equatorial crossing time of 10:30 am), with the exception of ice clouds over land in July. Figure 7 shows the zonal variation of $\chi$ from eq. (11) followed by monthly averaging for Terra and Aqua. Terra liquid clouds appear more homogeneous nearly everywhere for both months, except for northern midlatitudes, while ice clouds show fewer differences in the zonal distribution of $\chi$ between the two satellites; the major divergence of values (Terra clouds more homogeneous than Aqua clouds) occurs in the southern tropics in January (an area of convective activity) and in the mid-latitudes of the NH in July (again, Terra clouds more homogeneous).

\section{e. Winter vs. summer inhomogeneity}

Several of the figures shown thus far included results for both July and January, even though the contrast between these two months was not discussed. We will now revisit some of these figures to concentrate on seasonal differences. But first, we will look at the simplest, first order comparison, which is that among hemispheric averages. Figure 8 shows global and hemispheric averages of $\chi$ as derived from the D3 optical thickness moments of Terra. For liquid clouds the global values of these two parameters are virtually identical for both months while the winter and summer values are in almost perfect seasonal correspondance: northern hemisphere $(\mathrm{NH})$ winter equals southern hemisphere (SH) winter and $\mathrm{NH}$ summer matches $\mathrm{SH}$ summer. For ice clouds there are slight deviations from this behaviour: clouds are slightly less inhomogeneous on a global basis for $\mathrm{NH}$ winter; $\mathrm{NH}$ winter matches $\mathrm{SH}$ winter, but $\mathrm{NH}$ summer has more 
inhomogeneous clouds than SH summer. Overall, clouds are more inhomogeneous during the winter for both liquid and ice clouds.

Figure 6 provides information on seasonal contrast of global cloud variability separately for land and ocean and the two platforms. For liquid clouds, the global values of $\chi$ are the almost the same for the two months when the comparison is restricted to the same platform, and this is true for both marine and continental clouds. This is consistent with the liquid cloud results of Fig. 8. For ice clouds there are greater differences, with land clouds being more homogeneous for $\mathrm{NH}$ summer for both platforms; on the other hand, ocean clouds are more homogeneous for $\mathrm{NH}$ winter.

Finally, Fig. 7 shows the decreasing trend of liquid cloud homogeneity as one moves from northern to southern latitudes in July; the opposite takes place in January. The July and January values of $\chi$ meet around the Tropics, but then diverge in the midlatitudes with July heterogeneity being greater (smaller $\chi$ values) in the SH and January heterogeneity being greater in the NH. Same mid-latitude behavior can be discerned for ice clouds. For the ice phase, however, there is a trough of local heterogeneity maximum in the Tropics which highlights the contrast of cloud types which are identified as having ice particles between the Tropics (deep convective clouds) and summer mid-latitudes (cirrus). Still, the ice clouds of winter mid-latitude storm systems (stratiform precipitating clouds) appear more heterogeneous than the convective clouds of the Tropics.

\section{f. Geographical distribution of inhomogeneity}

In this section we examine regional characteristics of the inhomogeneity parameters that briefly touch on the behaviour of individual cloud regimes. Only the major features are 
discussed here with detailed analysis of specific regions with a multi-month dataset forthcoming in a separate study. The maps of inhomogeneity parameter $\chi$ shown and discussed here (subsection iii) can be compared with similar maps from ISCCP provided separately for low, middle and high clouds in http://isccp.giss.nasa.gov. The latter are actually expressed in terms of the inhomogeneity parameter $\varepsilon$, which is approximately, and under certain conditions (as discussed in section 2), related to $\chi$ via $\varepsilon \approx 1-\chi$. But even if the relationship between the two parameters was exact, direct comparison of these maps should not be done without exercising caution: there are differences in the grid on which the parameters are evaluated $\left(1^{\circ} \times 1^{\circ}\right.$ for MODIS, $\sim(280 \mathrm{~km})^{2}$ for ISCCP), the pixel size of the original retrievals ( $1 \mathrm{~km}$ for MODIS, $\sim 5 \mathrm{~km}$ for ISCCP), the sampling scale ( $\sim 5 \mathrm{~km}$ for MODIS, $\sim 30 \mathrm{~km}$ for ISCCP). Other distinguishing factors exist as well, such as different temporal sampling (sun-synchronous vs. geostationary), different cloud retrieval methods. etc. But before the ISCCP-MODIS comparison, we reexamine the zonal distribution of $\chi$ and identify the first-order contrast between marine and continental clouds.

\section{i. ZoNAL Distribution}

Figure 9 shows once again the zonal distribution of monthly values of $\chi$, but this time its correlation with the zonal variations of mean optical thickness and cloud fraction is also examined. Differences in zonal structure between July and January have already been discussed.

For liquid clouds, there does not seem to be any particular correlation between $\chi$ and either of the other two cloud parameters. Mean optical thickness increases almost 
monotonically as one moves away from the equator both northward and southward, but such symmetry is not observed for $\chi$ : latitude zones with similar values of mean $\tau$ can have very different values of $\chi$, indicating the presence of different cloud types. Winter clouds are more inhomogeneous than summer clouds for similar mean optical thicknesses, indicating wider distributions of $\tau$. Whether the low winter illumination angle is a contributor to the apparent increase of $\tau$-PDF width, through systematic effects on cloud retrievals remains an open question. There seems to be no clear correlation of $\chi$ with cloud fraction either. Still, for January, when the cloud fraction of liquid clouds shows a more robust zonal structure, the general tendency of SH clouds to be more homogeneous goes along with higher cloud fractions as in the marine boundary layer clouds of Barker et al. (1996).

Mid-latitude winter ice clouds, similarly to liquid clouds, tend to be more inhomogeneous than mid-latitude summer clouds. The local tropical minimum in $\chi$ (maximum inhomogeneity) shifts from $\sim 10^{\circ} \mathrm{S}$ in July to $\sim 10^{\circ} \mathrm{N}$ in January (where it is less well defined). It does not therefore coincide with the location of the Intertropical Convergence Zone (ITCZ) which can be coarsely identified as the local tropical maximum in cloud fraction and mean $\tau$. For the ITCZ the zonal monthly-averaged $\chi$ values are in the neigborhood of 0.7 (in general agreement with the high-cloud results of RDC). Contrary to liquid clouds, increased homogeneity for mid-latitude summer clouds is apparently accompanied by decreases in cloud fraction.

Overall, for zonally and monthly-averaged data, cloud inhomogeneity does not appear to correlate well with neither mean $\tau$, nor cloud fraction. Similar results were derived for gridpoint (non-zonal average) data, with or without monthly averaging. This 
picture is largely consistent with the results of RDC (their figures 13 and 14) and indicates that simple parameterizations of inhomogeneity parameters for LSMs, applicable to all cloud types, are not supported by the observations.

\section{ii. LAND VS. OCEAN}

We performed global and zonal averages separately for land and ocean pixels. $1^{\circ} \times 1^{\circ}$ coastal gridpoints (i.e. gridpoints that contained both ocean and land surfaces) were excluded from these calculations. Figure 6 indicates that liquid clouds are more variable over ocean than over land (as was found by RDC) while for ice clouds the results are inconclusive with one month (January) having less variable ocean clouds and the other (July) exhibiting the opposite behaviour. The bias in ice optical thickness retrievals over land mentioned in section 3 (no ice cloud optical thickness below 1) probably affects these results to some degree. The ocean-land contrast for liquid clouds is particularly strong in the mid-latitudes of the NH in winter, according to the top panel of Fig. 11. The bottom panel of the same figure shows that the much stronger homogeneity of continental ice clouds in the Tropics and subtropics is reversed at mid- and high latitudes.

\section{iii. Cloud Systems}

Plates 1 and 2 show the full geographical distribution of $\chi$ as derived with eq. (10) from Terra July '03 and Jan'04 optical thickness moments. The marine stratocumulus regimes off the west coasts of N. America, S. America, south-central Africa appear rather homogeneous in these maps with values of $\chi$ greater than $\sim 0.85$ in July and above $\sim 0.8$ in January, consistent with the satellite values of Pincus et al. (1999). These values are, 
however, larger than $\sim 0.7$ and $\sim 0.6$ found by Cahalan et al. (1994) and Cahalan et al. (1995) for FIRE (First ISCCP Regional Experiment) and ASTEX (Atlantic Stratocumulus Transition Experiment) marine boundary layer clouds, but which were based on different measurements (one or half minute-averaged microwave radiometer ground measurements) resolving temporal and not spatial (as in MODIS) variations. RDC finds annual mean values of $\varepsilon$ between 0.1 and 0.2 for these cloud systems which correspond to an approximare range of 0.8-0.9 for $\chi$. The region of active convection over Indonesia (ice cloud panels) appears to be characterized by $\chi$ values close to 0.6 (annual $\varepsilon$ between 0.3 and 0.4 in RDC). The same approximate value apparently characterizes the mid-latitude storm systems of both winter hemispheres. The ice clouds of the summer hemispheres are quite homogeneous with values of $\chi$ above 0.8 in most regions; the annual values of $\varepsilon$ in RDC for mid-latitude high clouds are between 0.1 and 0.2 for both hemispheres. Comparison with the ISCCP results of RDC is not straighforward for various reasons previously mentioned, but also because of the different cloud-type classification. Despite this, a first-order ISCCP-MODIS comparison for the two months of our analysis can be made by visual inspection of maps of $\varepsilon$ in the ISCCP website. The figures given there have a lot of the qualitative features of Plates 1 and 2 .

\section{Discussion and Conclusions}

This paper describes the first extensive attempt to infer horizontal inhomogeneity of cloud optical properties from the MODIS instrument aboard Terra and Aqua. Various options to derive cloud variability parameters from MODIS data are presented and the foundation for a climatology of cloud heterogeneity is layed with calculations of monthly 
inhomogeneity parameter values at $1^{\circ} \times 1^{\circ}$, zonal, and global spatial scales. Two full months (January and July 2003) of MODIS Atmosphere Level-3 data are analyzed. Geographical, diurnal, and seasonal changes of cloud optical property variability are studied, and the dependence on phase is examined.

We find that cloud inhomogeneity is weaker in summer than in winter, weaker over land than ocean (mainly for liquid clouds), weaker for local morning (Terra) than local afternoon (Aqua), about the same for liquid and ice clouds on a global scale, but with wider probability distribution functions (PDFs) and larger latitudinal variations for ice, and relatively insensitive to whether water path or optical thickness distributions are used. Typical mean monthly values at global and hemispherical scales of the inhomogeneity parameter $v$ (roughly the mean over standard deviation of water path or optical thickness), range from $\sim 2.5$ to 3 , while for the inhomogeneity parameter $\chi$ (the ratio of the logarithmic to linear mean), which is the centerpiece of our presentation, from $\sim 0.7$ to 0.8 . Zonal monthly values of $\chi$ can in some cases fall below 0.6 while monthly values of individual gridpoints can occasionally assume values below 0.5 . No obvious correlation between cloud inhomogeneity and cloud fraction or mean optical thickness was found. Finally, there is broad agreement in the geographical distribution of inhomogeneity parameter values between MODIS and ISCCP despite inherent differences between the two datasets in many aspects of the cloud retrievals and the parameter calculations.

These results show that MODIS is able to reveal the great richness in the variability of cloud horizontal inhomogeneity and underlines the fact that calculation of the global radiative effect of cloud water variations amount to more than a "back-of-the-envelope" 
calculation that uses the global mean of an inhomogeneity parameter. It is obvious that this paper is not the final word on the use of MODIS for studying cloud variability. Subject to funding, we plan to fine-tune our analysis method and extend the work to all available months of Terra and Aqua observations. With the multi-month analysis, features of cloud inhomogeneity detected here (land-ocean, morning-afternoon, liquidice, and seasonal contrasts) will be further explored, and potentially interpreted as consequences of the atmospheric state and cloud microphysical processes.

The non-linear dependence of solar and longwave radiation on cloud optical properties has to be taken into account for accurate calculations of the radiative energy budget. This work, along with that by Rossow et al. (2002), has reaffirmed that sufficient observations currently exist to achieve this on a global basis. One way to accomplish this is to provide Column Radiation Models with cloud water inhomogeneity information from MODIS along with modelled cloud vertical variability based on information from Cloud Resolving Models and future observations by CloudSat (Stephens et al., 2002). Such a study is part of our future plans.

Acknowledgements: The financial support of NASA under grant NAG5-11631 and of DoE's ARM program under grant DE-AI02-00ER62939 is greatfully acknowledged. We would like to thank Steve Platnick for his invaluable assistance in understanding various aspects of the MODIS cloud products. Also thanks go to Paul Hubanks and William Ridgway for their help with software and other technical issues. 


\section{References}

Barker, H. W., 1996: A parameterization for computing grid-averaged solar fluxes for inhomogeneous marine boundary layer clouds, part I: methodology and homogeneous biases. J. Atmos. Sci., 53, 2289-2303.

Barker, H. W., B. A. Wielicki, L. Parker, 1996: A parameterization for computing gridaveraged solar fluxes for inhomogeneous marine boundary layer clouds, part II: validation using satellite data. J. Atmos. Sci., 53, 2304-2316.

Cahalan, R. F., W. Ridgway, W. J. Wiscombe, T. L. Bell and J. B. Snider, 1994: The albedo of fractal stratocumulus clouds. J. Atmos. Sci., 51, 2434-2455.

Cahalan, R. F., D. Silberstein, and J. B. Snider, 1995: Liquid water path and planeparallel albedo bias during ASTEX. J. Atmos. Sci., 52, 3002-3012.

Harshvardhan, and D. A. Randall, 1985: comments on "The parameterization of radiation for numerical weather prediction and climate models". Mon. Wea. Rev., 113, 18321833.

Jakob, C. and S. A. Klein, 1999: The role of vertically varying cloud fraction in the parameterization of microphysical processes in the ECMWF model. Q. J. $R$. Meteorol. Soc., 125, 941-965.

King, M. D., W. P. Menzel, Y. J. Kaufman, D. Tanré, B.-C. Gao, S. Platnick, S. A. Ackerman, L. A. Remer, R. Pincus, and P. A. Hubanks, 2003: Cloud and aerosol properties, precipitable water, and profiles of temperature and water vapor from MODIS. IEEE Trans. Geosc. Rem. Sens., 41, 442-458. 
Oreopoulos, L., and R. Davies, 1998a: Plane parallel albedo biases from satellite observations. Part I: Dependence on resolution and other factors. J. Climate, 11, 919-932.

Oreopoulos, L., and R. Davies, 1998b: Plane parallel albedo błases from satellite observations. Part II: Parameterizations for bias removal. J. Climate, 11, 933-944.

Oreopoulos, L., and H. W. Barker, 1999: Accounting for subgrid-scale cloud variability in a multi-layer $1 \mathrm{~d}$ solar radiative algorithm. Q. J. Roy. Met. Soc., 126, 301-330

Oreopoulos, L., R. F. Cahalan, A. Marshak, and G. Wen, 2000: A new normalized cloud retrieval technique applied to Landsat radiances over the Oklahoma ARM site. $J$. Appl. Meteor., 39, 2305-2321.

Oreopoulos, L., 2004: The impact of subsampling on MODIS Level-3 statistics of cloud optical thickness and effective radius, IEEE Trans. Geosc. Rem. Sens., submitted.

Pincus, R., S. A. McFarlane, and S. A. Klein, 1999: Albedo bias and the horizontal variabiltiy of clouds in subtropical marine boundary layers: observations fron ships and satellites. J. Geophys. Res., 104, 6183-6191.

Platnick, S., M. D. King, S. A. Ackerman, W. P. Menzel, B. A. Baum, , J. C. Riédi, and R. A. Frey, 2003: The MODIS cloud products: Algorithms and examples from Terra. IEEE Trans. Geosc. Rem. Sens., 41, 459-473.

Räisänen, P., H. W. Barker, M. F. Khairoutdinov, J. Li, and D. A. Randall, 2004: Stochastic generation of subgrid-scale cloudy columns for large-scale models. $Q$. $J$. R. Meteor. Soc., in press. 
Räisänen, P., G. Isaac, H. W. Barker, and I. Gultepe, 2003: Solar radiative transfer for stratiform clouds with horizontal variations in liquid-water path and droplet effective radius. Q.J. R. Meteor. Soc., 129, 2135-2149.

Rossow, W. B., C. Delo, and B. Cairns, 2002: Implications of the observed mesoscale variations of clouds for the Earth's radiation budget. J. Climate, 15, 557-585.

Stephens, G. L., and coauthors, 2002: The CloudSat mission and the A-train. Bull. Amer. Meteor. Soc., 83, 1771-1790.

Tompkins, A. M., 2002: A prognostic parameterization for the subgrid-scale variability of water vapor and clouds in large-scale models and its use to diagnose cloud cover. J. Atmos. Sci., 59, 1917-1942.

Wilks, D. S., 1995: Statistical Methods in the Atmospheric Sciences, Academic Press. 467pp. 


\section{List of Tables}

Table 1 Conversion guide between $\chi$ and $v_{\text {MLE }}, v_{M O M}$ derived from Terra July and January zonal averages.

\begin{tabular}{|c|c|c|c|c|}
\hline$\chi$ & $v_{\text {MLE }}$ (liq) & $v_{\text {MOM }}($ liq) & $v_{\text {MLE }}$ (ice) & $v_{\text {MOM }}$ (ice) \\
\hline 0.50 & 1.03 & 0.85 & 1.07 & 0.96 \\
\hline 0.55 & 1.26 & 1.07 & 1.30 & 1.17 \\
\hline 0.60 & 1.54 & 1.34 & 1.56 & 1.43 \\
\hline 0.65 & 1.88 & 1.68 & 1.89 & 1.75 \\
\hline 0.70 & 2.30 & 2.10 & 2.29 & 2.14 \\
\hline 0.75 & 2.81 & 2.63 & 2.77 & 2.619 \\
\hline 0.80 & 3.43 & 3.29 & 3.34 & 3.19 \\
\hline 0.85 & 4.19 & 4.12 & 4.04 & 3.90 \\
\hline 0.90 & 5.12 & 5.17 & 4.89 & 4.76 \\
\hline 0.95 & 6.26 & 6.47 & 5.91 & 5.81 \\
\hline
\end{tabular}




\section{Figure captions}

Figure 1 Global monthly values of inhomogeneity parameters $\chi, v_{\text {MOM }}$, and $v_{\text {MLE }}$ derived using different methods and datasets. Details in the text.

Figure 2 Scatterplots of $v_{\text {MLE }}$ and $v_{\text {MOM }}$ vs. $\chi$ along with exponential fits for zonal averages (Terra, January and July). Top panel is for liquid clouds, bottom panel is for ice clouds.

Figure 3 Zonal-monthly (July and January) averages from Terra of $\chi$ for liquid (top) and ice (bottom) clouds as inferred from $\tau$ and $W$ histograms.

Figure 4 Zonal-monthly (July-top, January-bottom) averages from Terra of $\chi$ for liquid and ice clouds as inferred from $\tau$ moments.

Figure 5 Comparison of liquid phase and ice phase histograms of the monthly values of $\chi$ derived from eq. (10) for Terra. Top panel is for July and bottom panel for January.

Figure 6 Global land-only and global ocean-only monthly averages of $\chi$ for Terra and Aqua. Top panel is for liquid clouds and bottom panel is for ice clouds.

Figure 7 Zonal-monthly averages of $\chi$ for Terra and Aqua. Top panel is for liquid clouds and bottom panel is for ice clouds.

Figure 8 Global and hemispheric values of $\chi$ for July 2003 and January 2004 from Terra D3 optical thickness moments. Top panel is for liquid clouds and bottom panel is for ice clouds.

Figure 9 Zonal-monthly averages of $\chi, \tau$, and cloud fraction (CF) from Terra for both phases and both months analyzed.

Figure 10 Zonal-monthly averages of $\chi$ from Terra, calculated separately for land and ocean for both phases and both months. 
Plate 1 Geographical distribution of monthly averages of $\chi$ calculated from eq. (10) for Terra July 2003. Top panel is for liquid clouds and bottom panel is for ice clouds.

Plate 2 As in Plate 1, but for January 2004. 

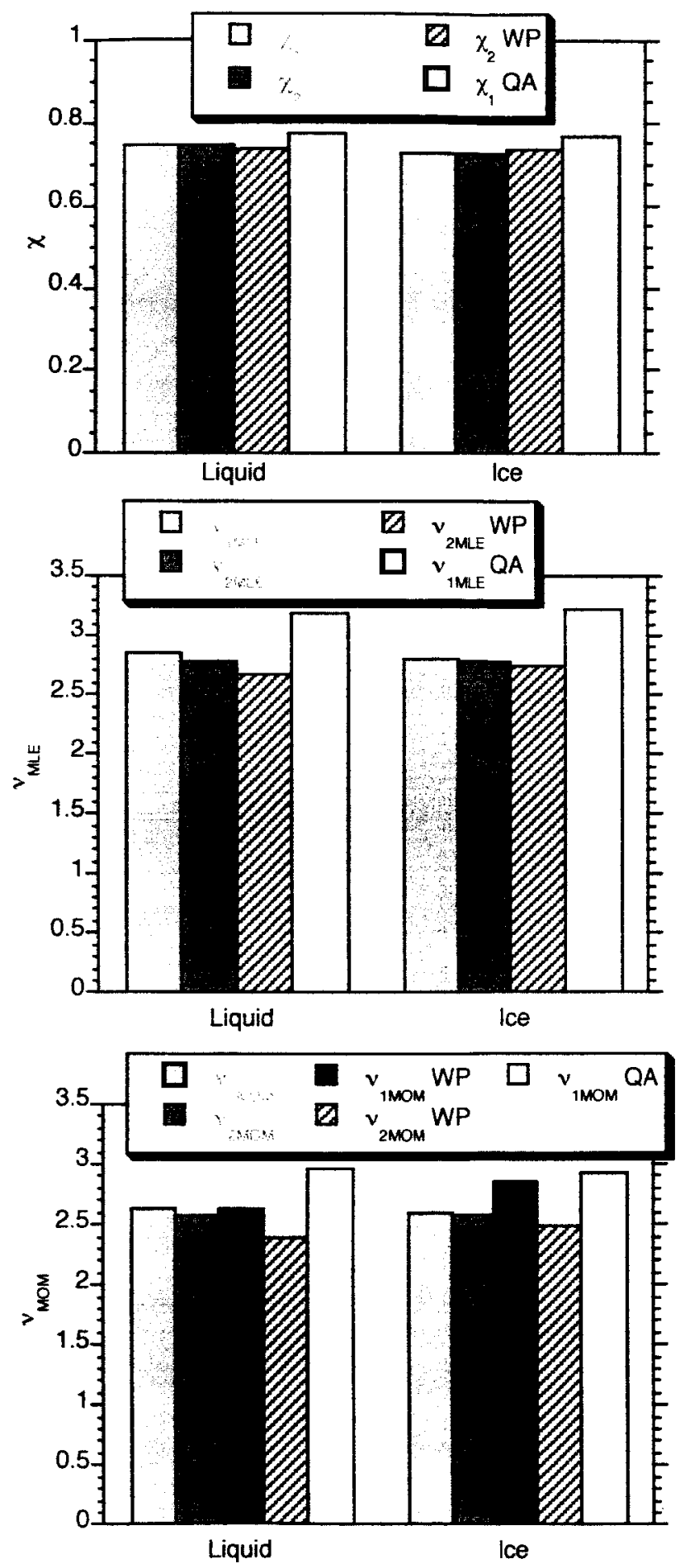

Figure 1 

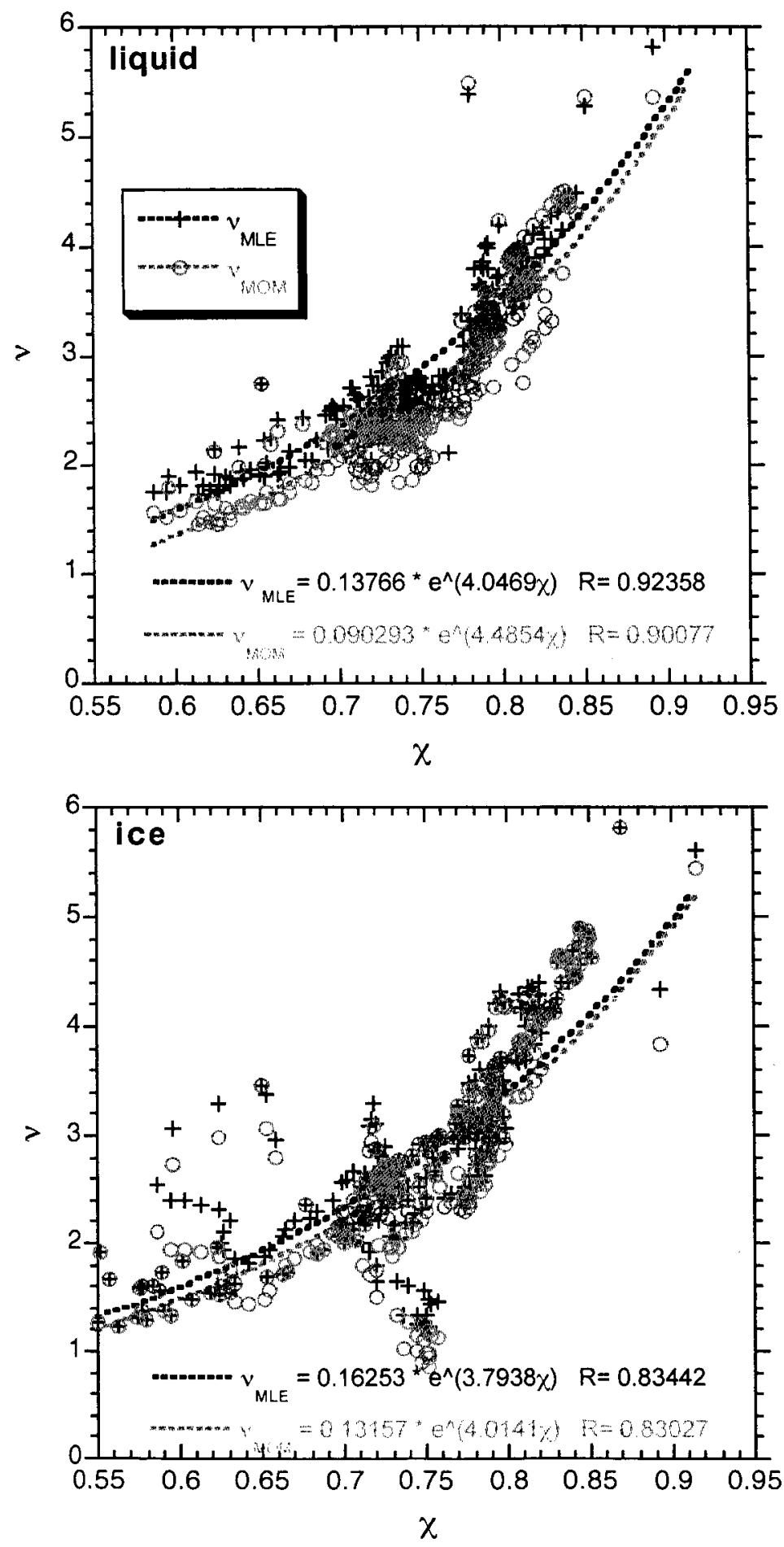

Figure 2 

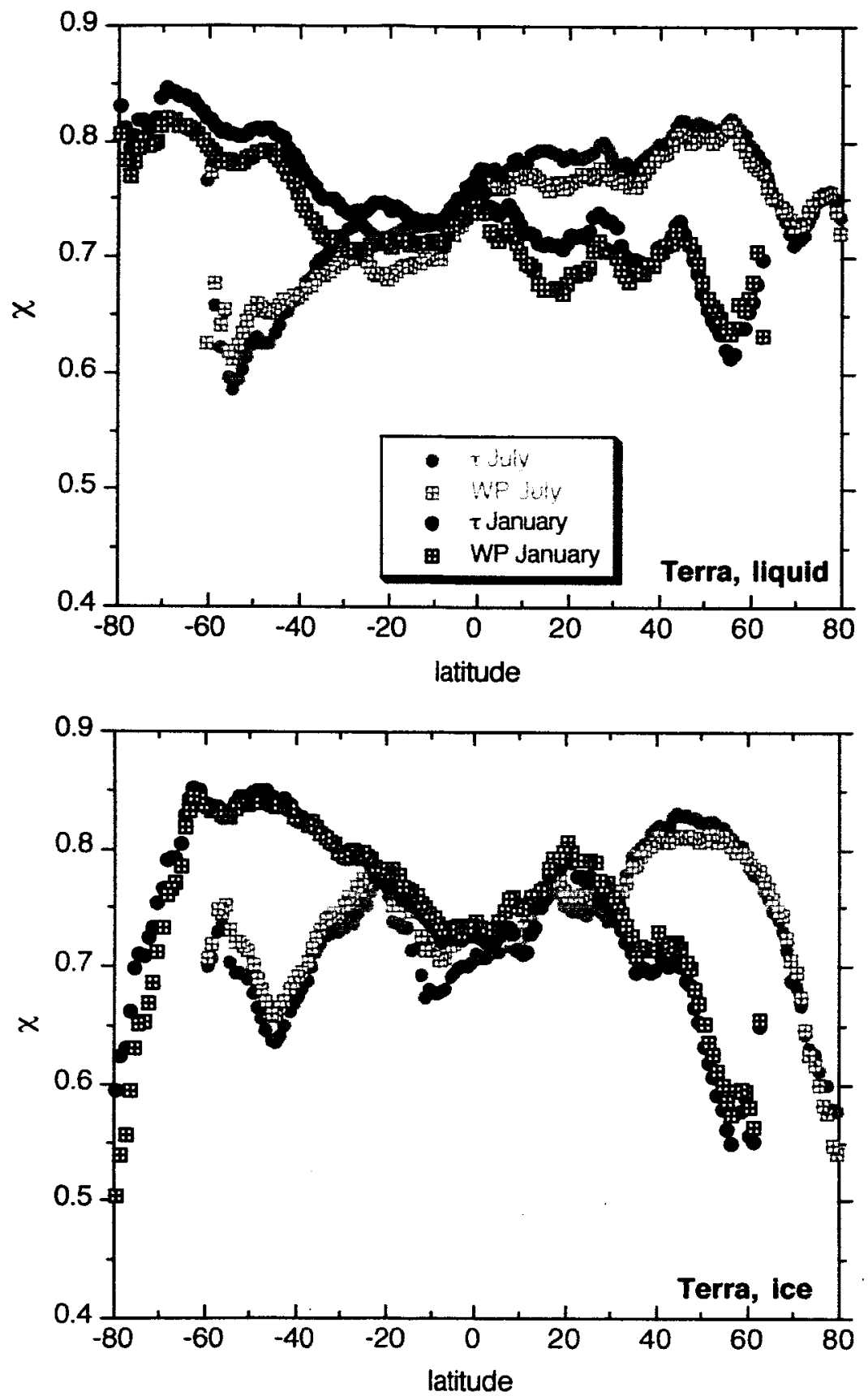

Figure 3 

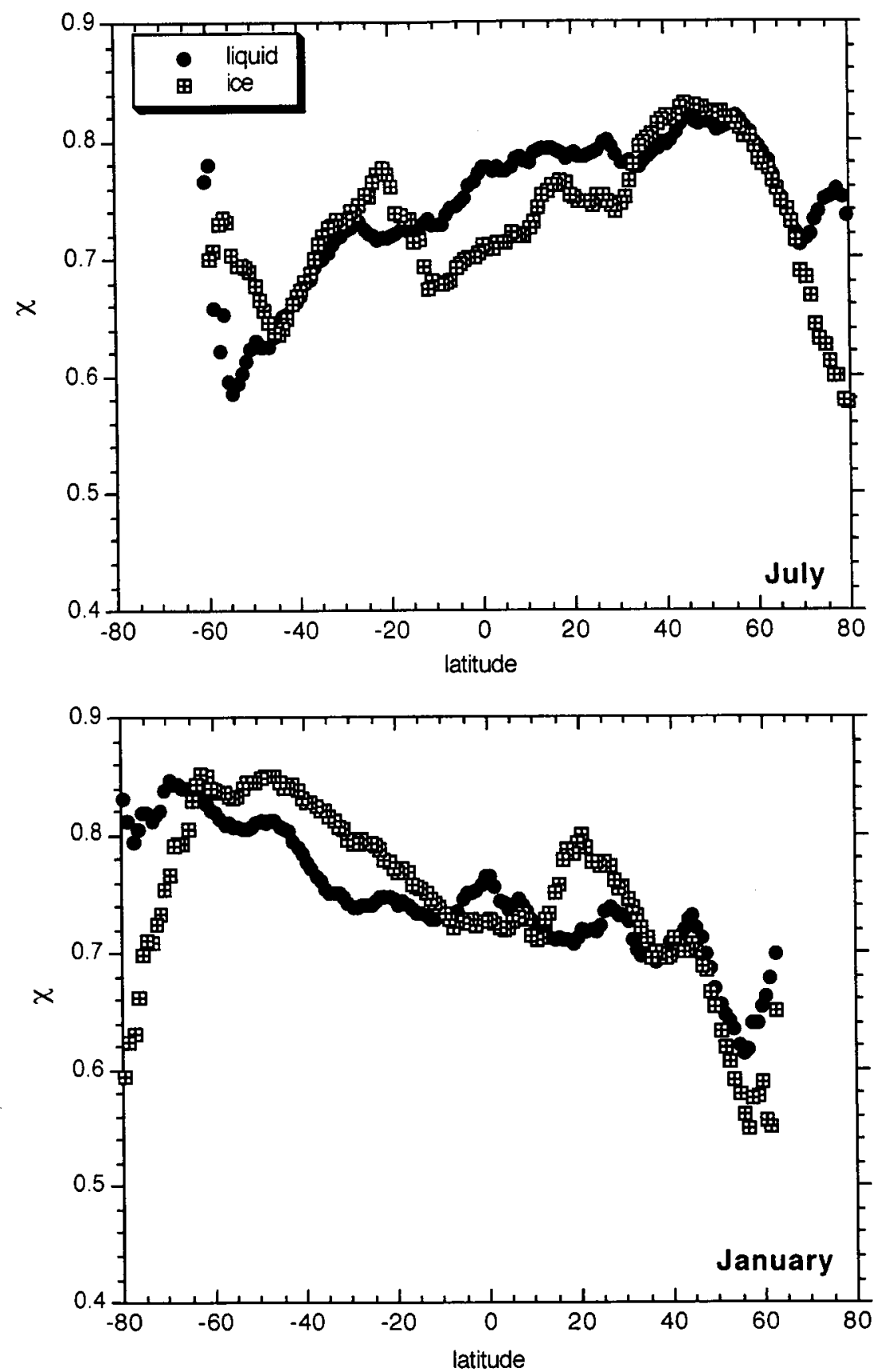

Figure 4 

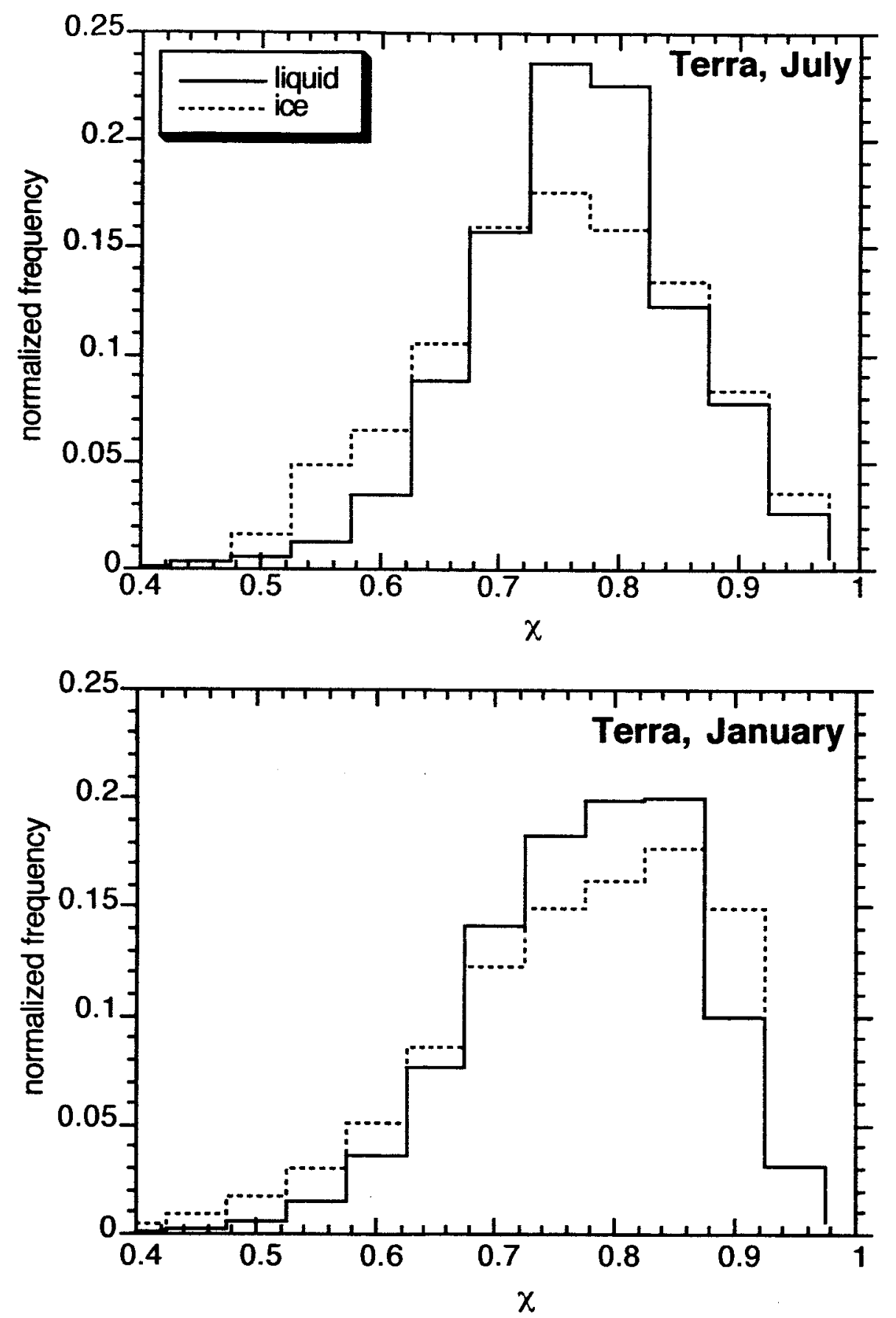

Figure 5 


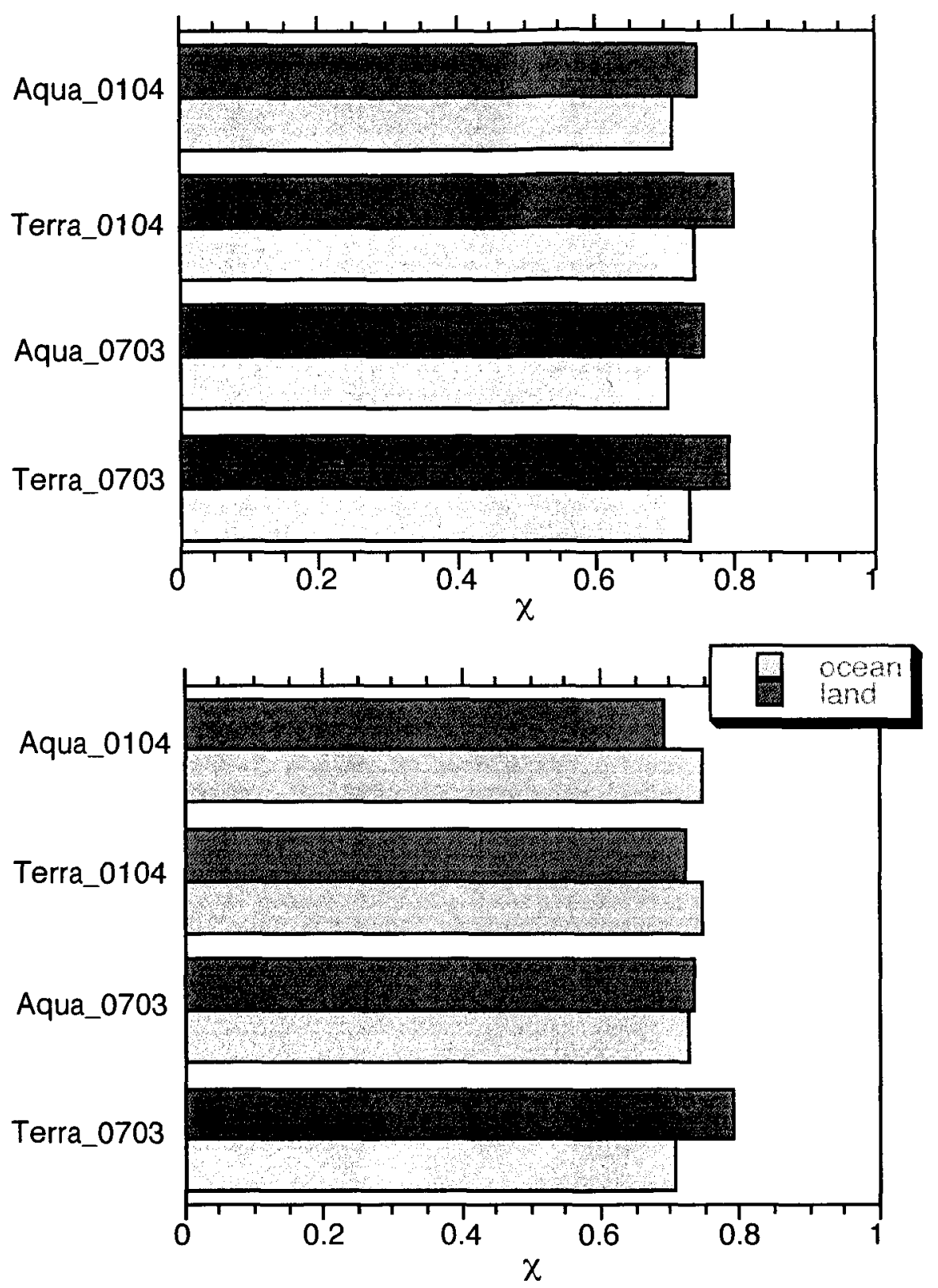

Figure 6 

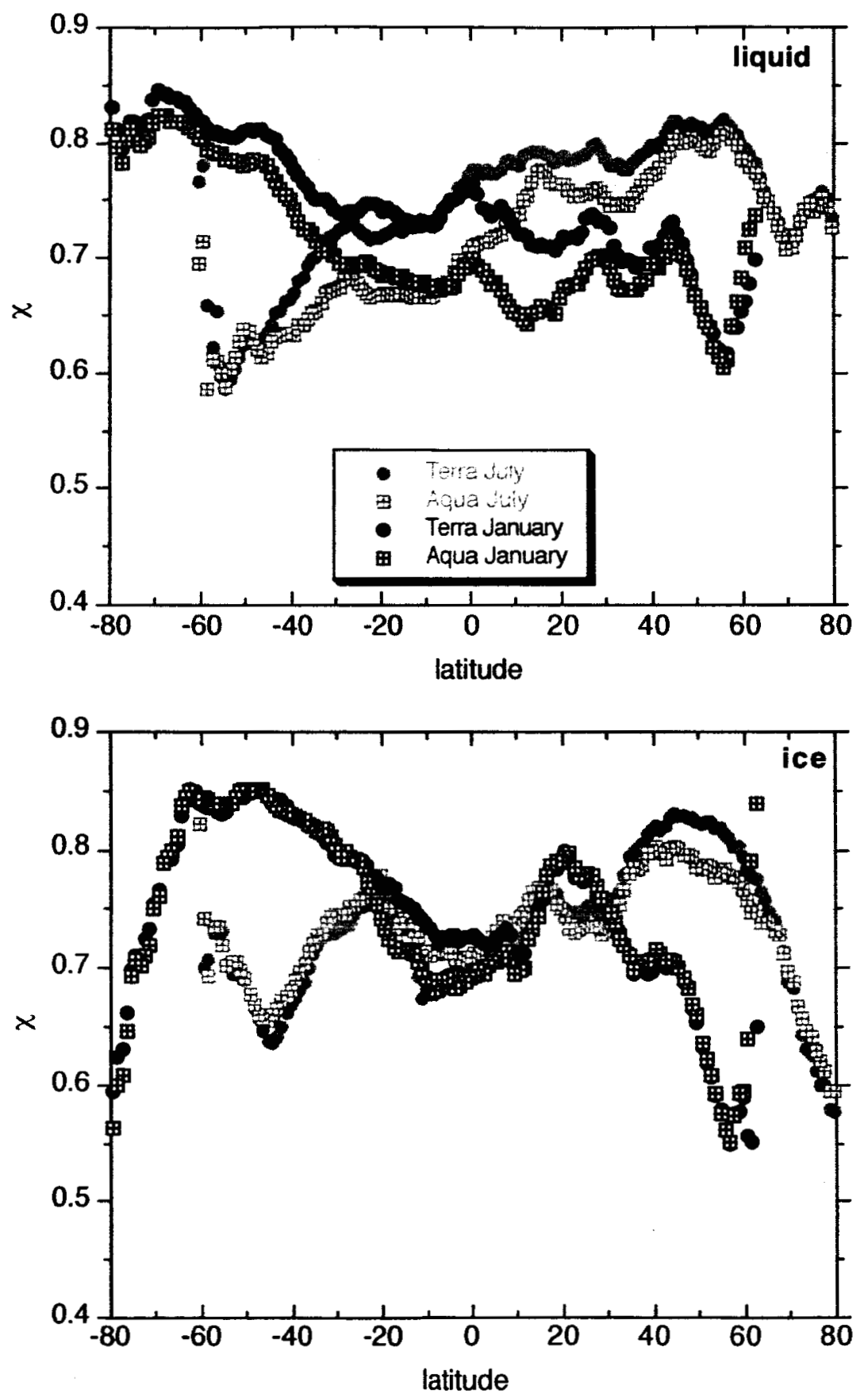

Figure 7 

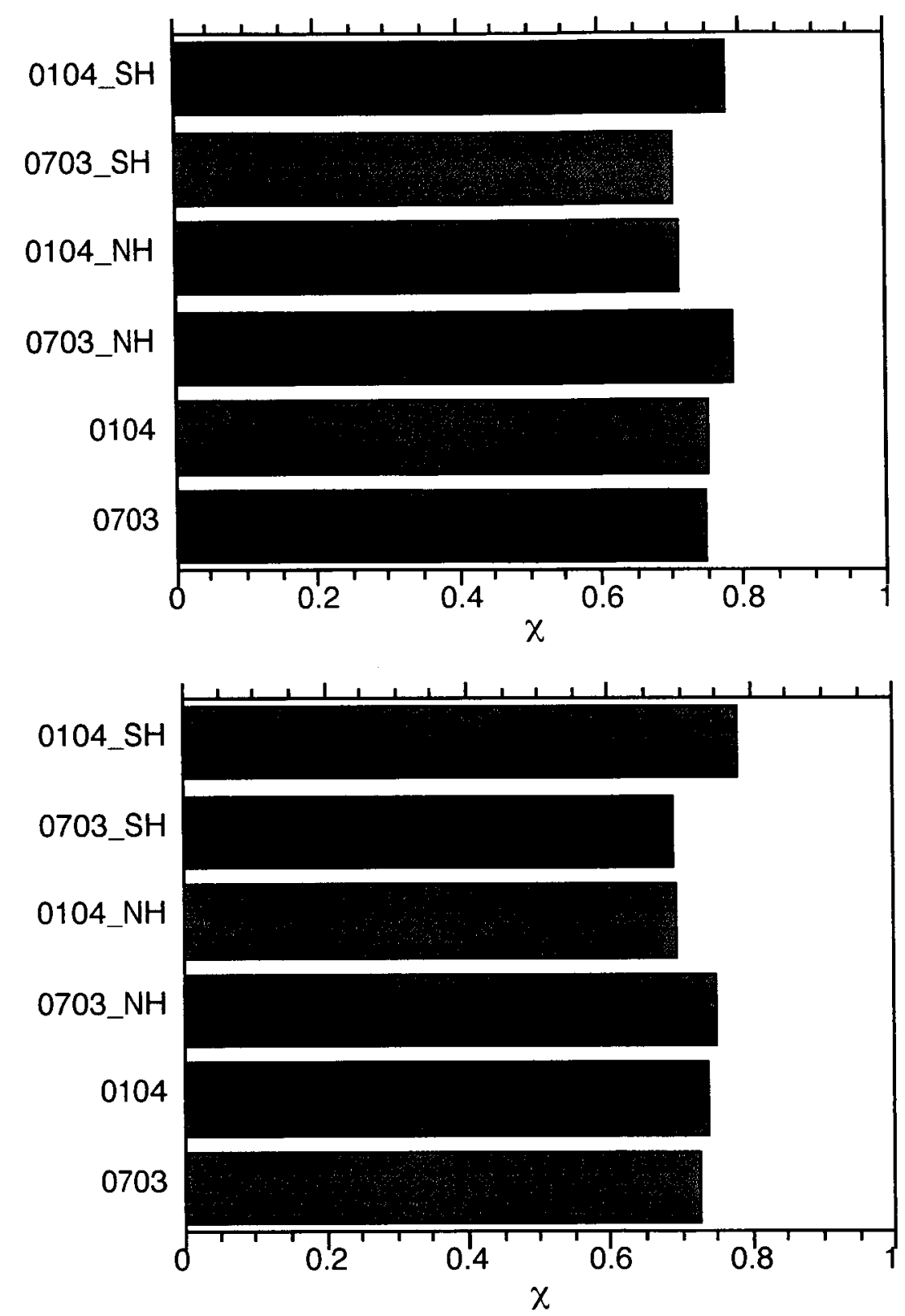

Figure 8 

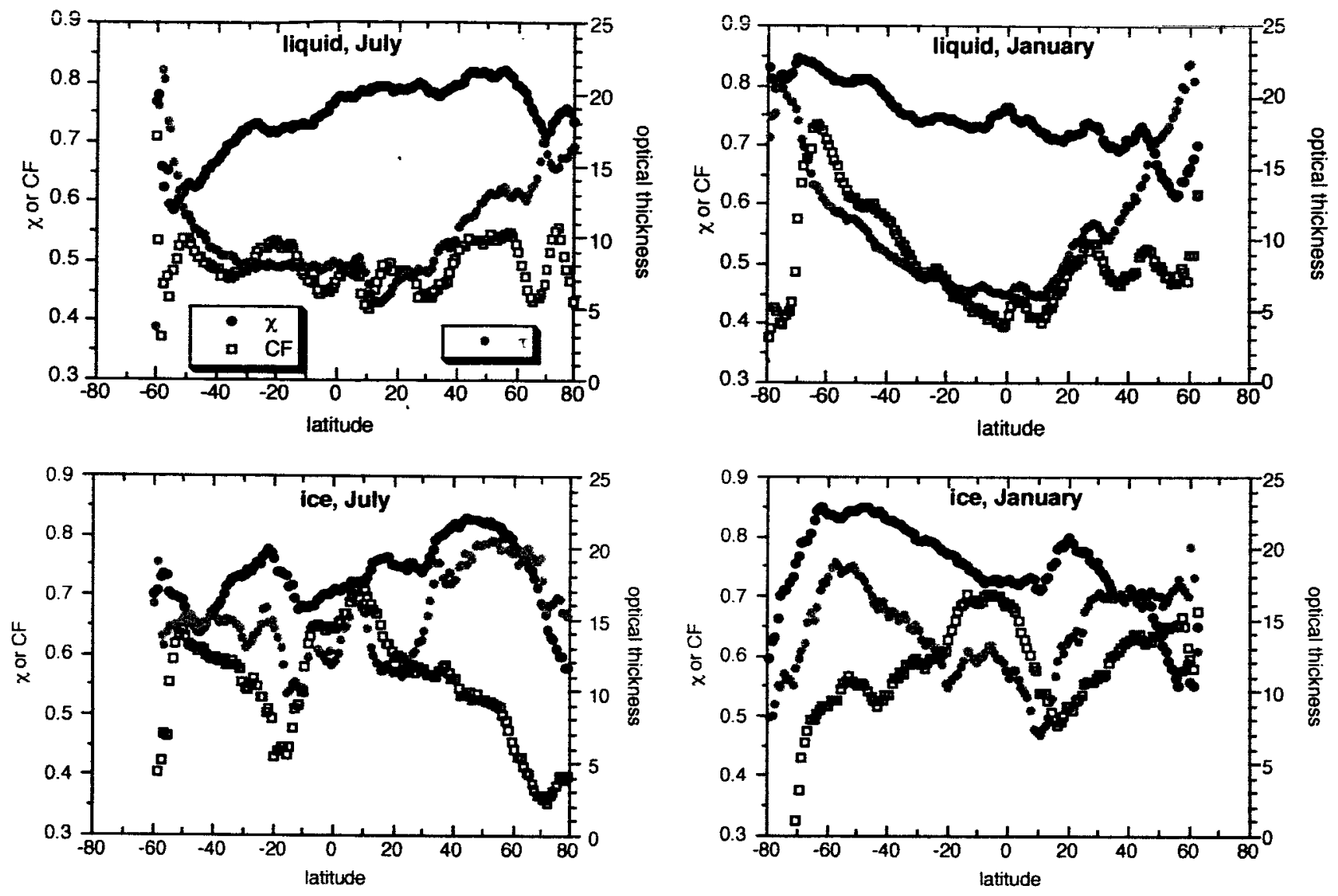

Figure 9 

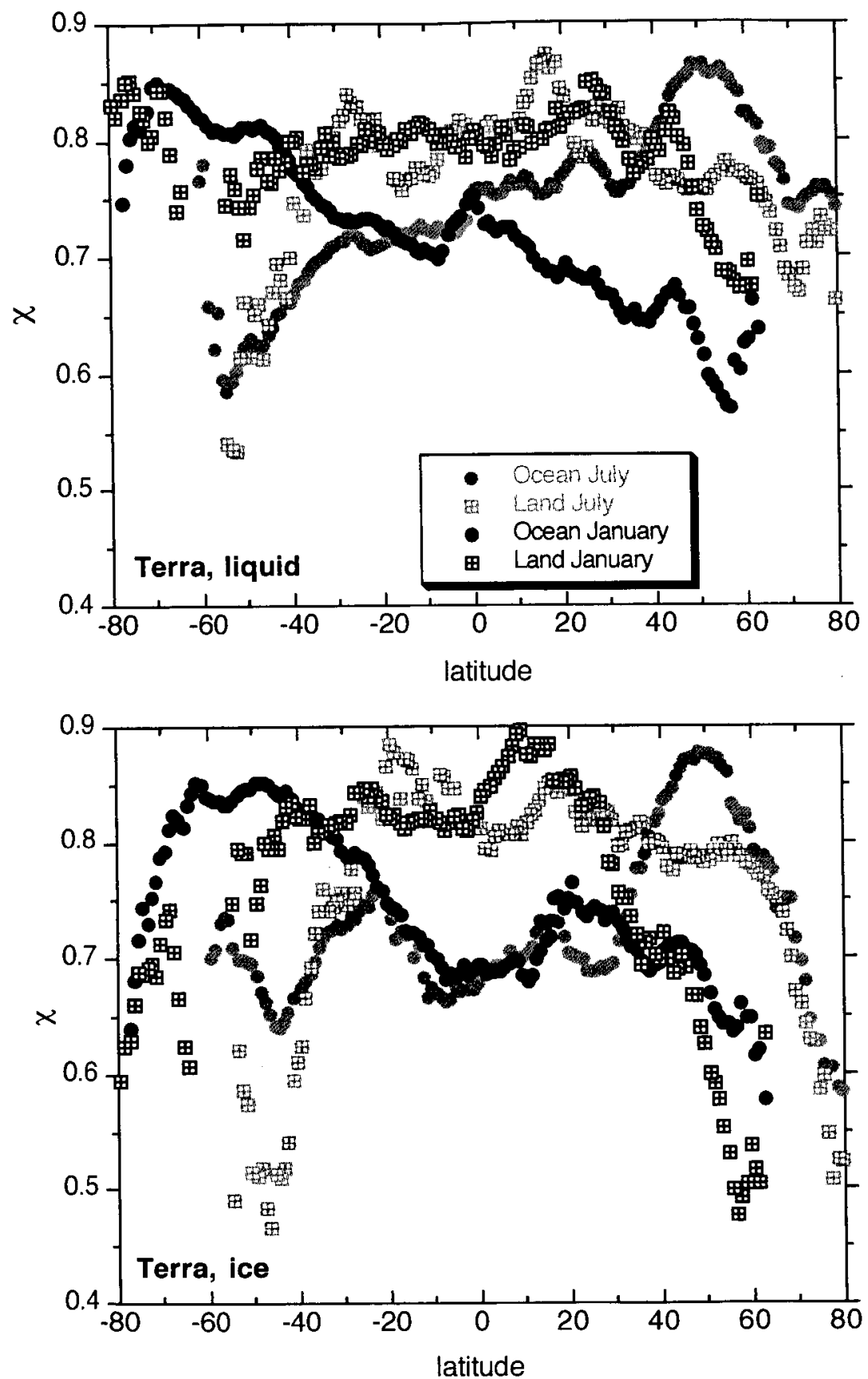

Figure 10 

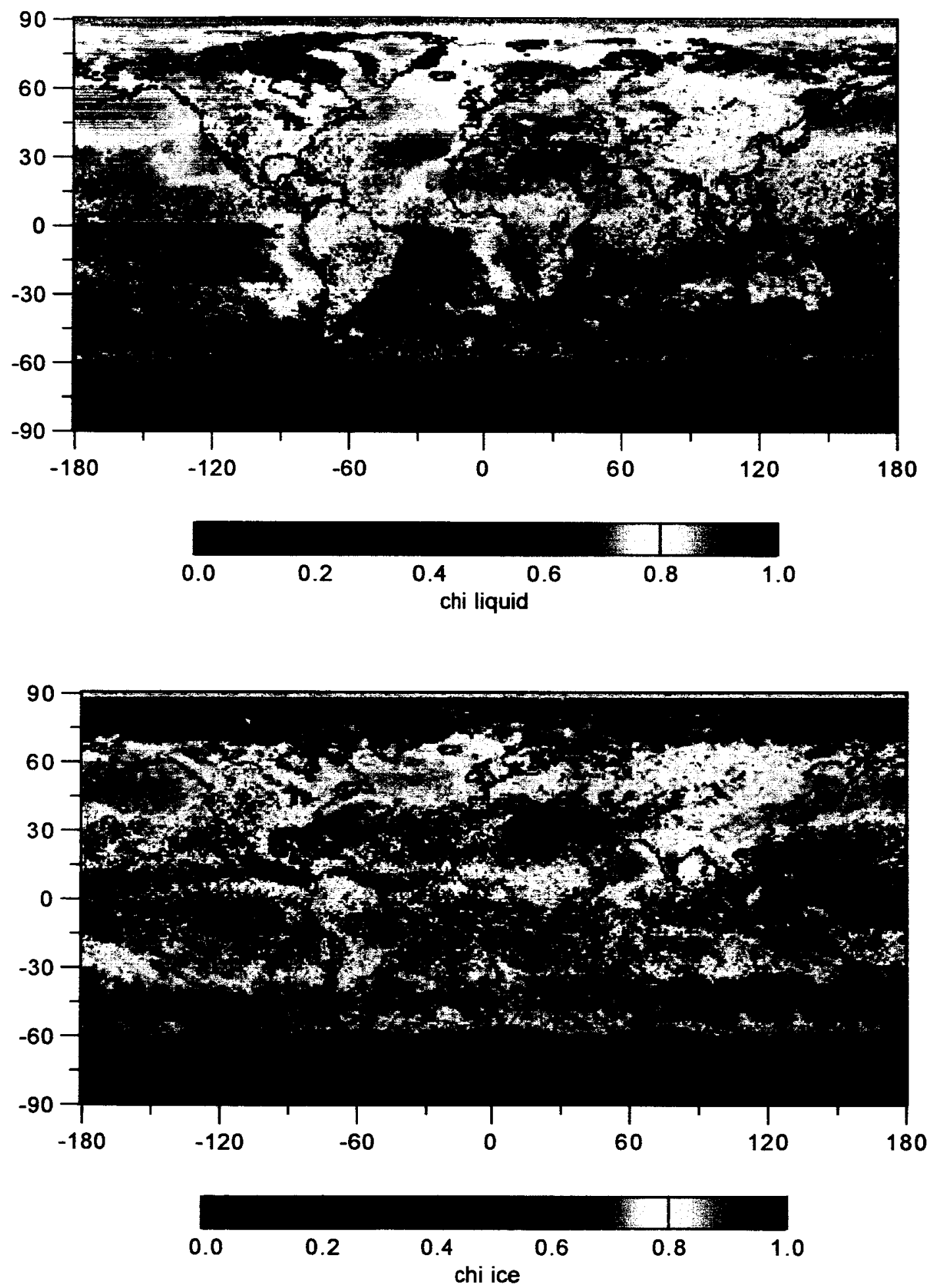

Plate 1 

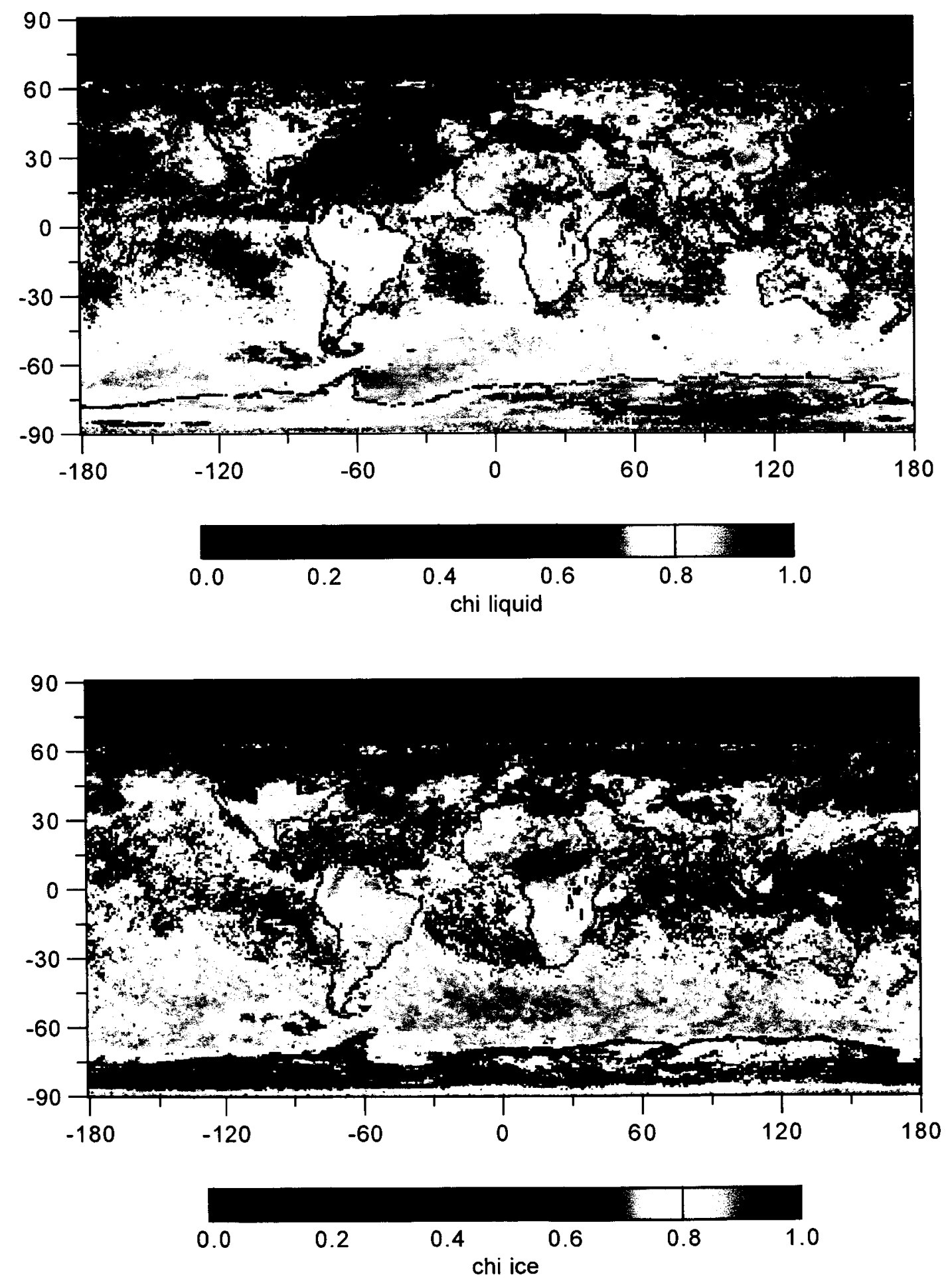

Plate 2 


\section{Popular summary for \\ "Cloud inhomogeneity from MODIS" \\ by Lazaros Oreopoulos and Robert F. Cahalan}

submitted to J. of Climate

This paper provides a global picture of cloud inhomogeneity at $\sim 100 \mathrm{~km}$ scales from 1 km observations by the instrument MODIS aboard the Terra and Aqua satellites. The reason we are interested in cloud inhomogeneity is because inhomogeneous clouds reflect, transmit, absorb, and emit different amounts of solar or thermal (infrared) radiation than their homogeneous counterparts. By measuring how much this inhomogeneity is on a global basis we hope that we can make advances in representing clouds of Global Climate Models more realistically. If the model clouds are not realistic, errors in the estimates of Earth's radiation budget are likely to occur. Assuming that one day we will be able to predict cloud variability in climate models, we would like to examine whether the variability of the models exhibits the same features as the variability in the observations. For example, our work finds that clouds are more variable over oceans than over land, more variable during the winter than the summer, more variable in the afternoon than in the morning. Will the models be able to reproduce these results? 